Hospital, Sydney, NSW, Australia; and ${ }^{3}$ Department of Surgery, St George Hospital, Sydney, NSW, Australia

Solid papillary carcinoma with reverse polarity is a rare breast cancer of specific morphologic and immunohistochemical profile with favourable prognosis. However, this carcinoma is not yet included in the current WHO classification. The tumour shows histologic features resembling tall cell variant of papillary thyroid carcinoma. We report a case of a 63-year-old lady with an incidental finding of a discrete oval mass on routine breast screening. Clinical and radiological findings are suggestive of a fibroadenoma. Histologically, the tumour shows papillary, follicular and solid structures. The cells are tall and columnar with abundant eosinophilic cytoplasm and apically located nuclei giving the impression of reverse nuclear polarity. Eosinophilic colloid like secretion is present. Nuclear pseudoinclusions and occasional grooves are also observed. Immunohistochemistry stains demonstrate the lack of myoepithelial cells, indicating its invasive nature. The tumour shows positive staining for cytokeratin $5 / 6$, calretinin and luminal staining for EMA. Thyroid marker, TTF1, is negative. Despite the indolent behaviour, the tumour is triple negative (negative ER, PR and HER2). Our findings demonstrate that solid papillary carcinoma with reverse polarity is a unique breast neoplasm that should be distinguished from other papillary carcinomas of the breast.

\section{DABIGATRAN-INDUCED OESOPHAGITIS DISSECANS SUPERFICIALIS}

Suneeth Mathew, Masato Yozu

Histopathology Department, Middlemore Hospital, Auckland, New Zealand

Dabigatran is a direct thrombin inhibitor frequently prescribed for prophylaxis of stroke in the setting of atrial fibrillation. The histologic features of dabigatran-induced drug injury have not been described in the literature. We describe herein five patients taking dabigatran, presenting with histologic features of oesophagitis dissecans superficialis.

The patients included three men and two women with a median age of 82 years (range 67-91 years). The clinical indications for taking dabigatran were atrial fibrillation $(80 \%)$ and deep vein thrombosis $(20 \%)$. Presenting symptoms included dysphagia (40\%), anaemia (40\%), nausea and vomiting (40\%), and epigastric pain (20\%). Endoscopically, epithelial sloughing typical of oesophagitis dissecans was seen in the mid or lower oesophagus in all patients. Histologically the oesophageal biopsies showed necrosis and splitting of the superficial squamous epithelium with preservation of the basal cell layer, characteristic of oesophagitis dissecans superficialis. This is the first report describing histologic features of dabigatran-induced drug injury in the gastrointestinal tract.

\section{REMOVED: ANTERIOR MEDIASTINAL CHOLESTEROL GRANULOMA - A CASE REPORT}

$\underline{\text { Bulungo Florah Mwilambwe }}^{1}$, Sonu Nigam ${ }^{1}$, Sylvio Provenzano

${ }^{1}$ Department of Anatomical Pathology, Pathology Queensland, Gold Coast University Hospital, Gold Coast, Qld, Australia; and ${ }^{2}$ Department of Cardiothoracic Surgery, Gold Coast University Hospital, Gold Coast, Qld, Australia

This article has been removed: please see Elsevier Policy on Article Withdrawal (https://www.elsevier.com/about/ourbusiness/policies/article-withdrawal).

This article has been removed at the request of the Authors and the Editor in Chief due to patient consent issues.

\section{CASE CATEGORIES FOR CERVICAL BIOPSIES AND EXCISIONS: CONSISTENT TERMINOLOGY TO SUPPORT THE NATIONAL CERVICAL SCREENING PROGRAM (NCSP)}

Marsali Newman $^{1}$, Meagan Judge ${ }^{2}$, Kerryn Ireland Jenkin ${ }^{1,3}$

${ }^{1}$ Department of Anatomical Pathology, Austin Health, Melbourne, Vic, Australia; ${ }^{2}$ Royal College of Pathologists of Australasia, Sydney, NSW, Australia; and ${ }^{3}$ Department of Clinical Pathology, Melbourne Medical School, University of Melbourne, Vic, Australia

Background: The RCPA Structured Reporting Protocol for Excisions and Colposcopic Biopsies Performed for the Diagnosis and Treatment of Pre-invasive Cervical Neoplasia was developed to provide consistent terminology for histological reporting, allowing for data capture and clear implementation of management guidelines within the NCSP.

Aims: To report distribution of cases, assess practical utility and potential issues associated with the use of diagnostic categories for reporting histological cervical specimens.

Methods: Reports from consecutive diagnostic cervical biopsies and excision specimens were retrospectively reviewed and categorised for the squamous, glandular, and 'other' components according to the RCPA Structured Reporting Protocol.

Results: All cases were able to be retrospectively categorised. In 418 cervical biopsies, squamous component: not identified $7.7 \%$, normal/benign $44.7 \%$, LSIL $17.7 \%$, possible HSIL $1.2 \%$, HSIL $26.1 \%$, and malignant $2.6 \%$; endocervical component: not identified $15.9 \%$, normal/benign $76.3 \%$, possible AIS $0.2 \%$, AIS $0.2 \%$, malignant $1 \%$. 'Other neoplastic lesion' in 6 biopsy cases. In 249 cervical excisions, squamous component: normal/benign $28.9 \%$, LSIL $9.7 \%$, HSIL $58.6 \%$, and malignant $2.4 \%$; endocervical component: not identified $0.4 \%$, normal/benign $92.8 \%$, AIS $4.8 \%$, malignant $0.4 \%$.

Conclusions: Use of diagnostic categories in cervical biopsies and excisions is readily achievable for pathologists allowing for clear management pathways for women participating in the NCSP. 